\title{
Influence of mismatch on the defects in relaxed epitaxial InGaAs/GaAs(100) films grown by molecular beam epitaxy
}

\author{
D. I. Westwood and D. A. Woolf \\ Department of Physics and Astronomy, University of Wales College of Cardiff, P.O. Box 913, \\ Cardiff $C F 23 Y B$, Wales, United Kingdom \\ A. Vila, A. Cornet, and J. R. Morante \\ LCCM Departament de Fisica Aplicada i Electrònica, Avda. Diagonal 645, Barcelona 08028, Spain
}

(Received 22 March 1993; accepted for publication 13 April 1993)

\begin{abstract}
Thick $(\sim 3 \mu \mathrm{m})$ films of $\operatorname{In}_{x} \mathrm{Ga}_{1-x}$ As grown on $\mathrm{GaAs}(100)$ substrates, across the whole composition range, have been examined by transmission electron microscopy and double-crystal $x$-ray diffraction. The results were compared with the observed growth mode of the material determined by in situ reflection high-energy electron diffraction in the molecular beam epitaxy growth system. The quality of the material degraded noticeably for compositions up to $x \sim 0.5$ associated with an increased density of dislocations and stacking faults. In contrast, improvements in quality as $x$ approached 1.0 were correlated with the introduction of an increasingly more regular array of edge dislocations.
\end{abstract}

\section{INTRODUCTION}

Over the past few years, considerable interest has been devoted to the growth of heterostructures utilizing latticemismatched epitaxial layers for both fundamental and device research purposes. ${ }^{1}$ Among these mismatched epitaxial systems, $\mathrm{In}_{x} \mathrm{Ga}_{1 \ldots}$ As/GaAs is one of the most studied because of its potential for applications in high-speed ${ }^{2,3}$ and optoelectronic devices. ${ }^{4,5}$

The major problem with the InGaAs/GaAs system is that the lattice mismatch between the $\operatorname{In}_{x} \mathrm{Ga}_{1-x}$ As and the GaAs layers (up to $\sim 7 \%$ for $x=1.0$ ) creates defects in the epitaxial material. However, for single $\operatorname{In}_{x} \mathrm{Ga}_{1}{ }_{-x} \mathrm{As}$ epilayers on GaAs the mismatch can be accommodated elastically if the thickness of the layer is less than a critical thickness for defect formation, ${ }^{6}$ which depends upon the In concentration (and therefore mismatch) in the epilayer. Unfortunately, many potential uses require high In concentrations and epilayers thicker than the critical thickness. Under such conditions, misfit dislocations will form. Although pure edge dislocations with Burgers vector lying in the plane of the heterointerface can accommodate the maximum elastic strain ${ }^{7}$ they are difficult to form. As a consequence most of the misfit dislocations observed at low InAs mole fractions are dislocations with Burgers vectors of $a_{0} / 2\langle 110\rangle$ at $60^{\circ}$ to the dislocation line, i.e., so-called $60^{\circ}$ mixed dislocations, which thread up into the epitaxial layer. The presence of these dislocations results in poor crystalline quality throughout the film and unacceptable surface morphology. In addition the optical and electrical quality of such films is adversely affected by these structural defects.

Another difficulty in achieving high structural quality is thought to be the change, beyond the critical thickness, from a two-dimensional (2D or layer-by-layer) to threedimensional (3D or Stranski-Krastanow) growth mode at large misfits. ${ }^{8,9}$ Three-dimensional growth is known to occur for mole fractions greater than $x \sim 0.2-0.45$ depending on the growth temperature, ${ }^{8,10,11}$ and has been thought undesirable because it creates structural distortions by introducing variations in the layer thickness and strain, leading to crystallographic defects (such as dislocations, stacking faults, and dislocation complexes ${ }^{12}$ ).

This view is, however, at odds with the observed characteristics of thick films grown over the whole composition range where it is found, for example, that InAs films grown on $\operatorname{GaAs}(100$ ) (misfit $\sim 7.2 \%$ ) are, surprisingly, of comparable quality, as measured by $x$-ray diffraction, with $\mathrm{In}_{0.1} \mathrm{Ga}_{0.9}$ As films (misfit $\sim 0.7 \%$ ) of the same thickness. ${ }^{11}$ It is therefore of importance to clarify the evolution of the nature of defects in relaxed films with alloy composition (or misfit). This should lead to a better understanding of the mechanisms that are involved, and result in better control over the quality of the epitaxial layers.

Although sevcral publications have addressed the change of the defect structure observed in $\operatorname{In}_{x} \mathrm{Ga}_{1-x}$ As when $x$ increases, these have usually either concentrated on InAs mole fractions of up to only $x=0.5$ or on very thin films. ${ }^{8,12-14}$ Therefore, further investigation on the origin and evolution of defects in thick films over the whole composition range is necessary. The aim of this work is to provide, by using transmission electron microscopy (TEM), experimental information over a large range of InAs mole fractions, i.e., $0.039<x<1$, and to correlate the data with double-crystal $x$-ray diffraction results.

\section{EXPERIMENTAL DETAILS}

All of the epilayers were grown using a VG SEMICON V8OH molecular beam epitaxy (MBE) system which has bcen described elsewhere. ${ }^{11}$ The growth conditions used were: a growth rate of $\sim 1 \mu \mathrm{m} / \mathrm{h}$, a growth temperature of $350 \pm 20^{\circ} \mathrm{C}$, a layer thickness of $2.8 \pm 0.2$ $\mu \mathrm{m}$, and an $\mathrm{As}_{4} /(\mathrm{Ga}+\mathrm{In})$ flux ratio of $\sim 0.7$. The substrates used were liquid-encapsulated Czochralski (LEC) grown, undoped, on axis $\operatorname{GaAs}(100)$ wafers with quoted 

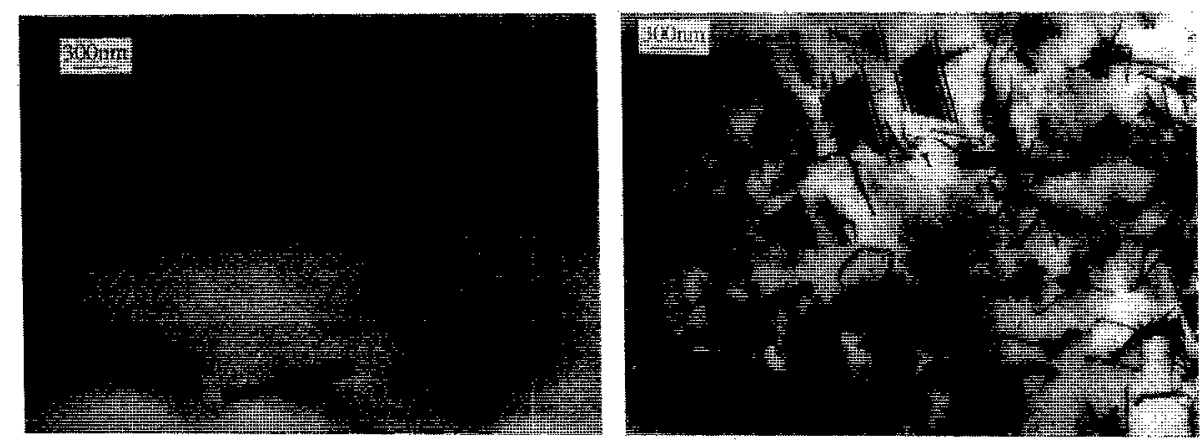

(c) $x=0.93$

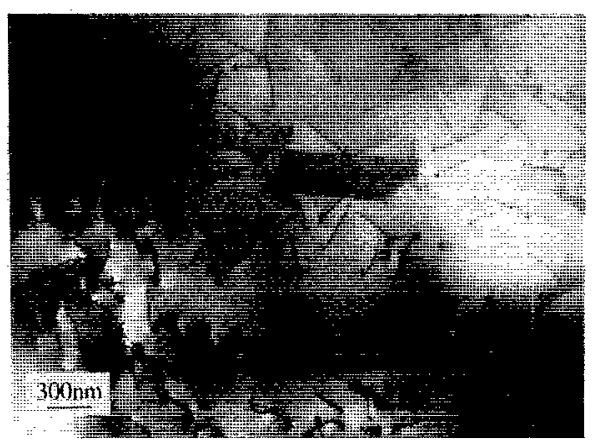

(d) $x=1.0$

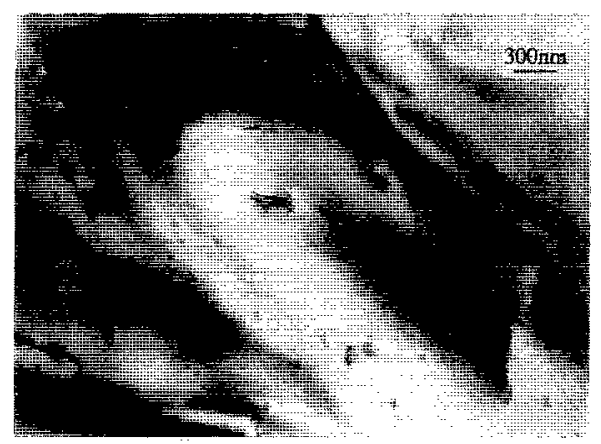

FIG. 1. Plan-view images of the near-surface dislocations in $\operatorname{In}_{x} \mathrm{Ga}_{1-x}$ As samples with (a) $x=0.04$, (b) $x=0.45$, (c) $x=0.93$, and (d) $x=1.0$. The single, slightly undulating, dark lines are threading dislocations, while the multiple closely spaced dark lines are stacking faults.

etch pit densities (epds) in the range (3-5) $\times 10^{5} \mathrm{~cm}^{-2}$. During growth the surface structure, and therefore the growth mode, were observed by reflection high-energy electron diffraction (RHEED).

Following growth, cross-section and plan-view [100] TEM specimens were prepared by mechanically polishing the substrate side to a thickness of $30 \mu \mathrm{m}$. A final thinning was carried out by ion milling on a cooled stage using low Ar ion intensities until the area of interest became thin enough for TEM investigation. The observations have been performed using a Hitachi H-800 NA microscope operating at $200 \mathrm{keV}$. Measurements with double-crystal x-ray diffraction (DCXRD) were also realized using a Bede 150 diffractometer, $\mathrm{Cu} K \alpha_{1}$ radiation and an InP (100) first crystal. These not only gave alloy compositions but also an indication of the material quality, through the width of the diffraction peaks.

\section{RESULTS}

The variation in the material quality across the whole composition range is illustrated in Fig. 1, which shows representative plan-view images of the surfaces of the samples for the compositions: (a) $x=0.04$, (b) $x=0.45$, (c) $x=0.93$, and (d) $x=1.0$. It is clear that the quality is worst, with large quantities of stacking faults and threading dislocations, for compositions around $x-0.5$. This is emphasized in Fig. 2, which shows the dependence of the surface density of threading dislocations (obtained by averaging values from a number of micrographs) as a function of the InAs alloy mole fraction, $x$. The stacking fault densities show a similar trend although with densities always at least an order of magnitude lower.

The improvement as $x$ decreases from 0.5 is not surprising considering the reduced mismatch, however, the similar behavior as $x$ goes to 1.0 is more unexpected. This behavior is confirmed in cross-sectional observations of the interfaces of the $x=0.45$ and 1.0 samples shown in Fig. 3 . While the $x=0.45$ sample shows a plethora of threading dislocations that extend into the InGaAs epilayer from the interface, the $x=1.0$ sample shows much fewer with most being confined near to the interface.

The dominant dislocations for mole fractions around $x=1.0$ are edge dislocations and these are shown imaged at the interface of the InAs/GaAs sample in Fig. 4. The image shows a reasonably regular network of dislocations. In contrast, similar images for the $x=0.93$ and 0.85 samples indicated arrays which became progressively less ordered as $x$ was decreased, It was not possible to image such arrays for $x$ values less than 0.85 .

DCXRD data displaying the full width at half maximum (FWHM) of the epitaxial layer peak plotted as a function of the InAs mole fraction $(x)$ are shown in Fig. 5. 


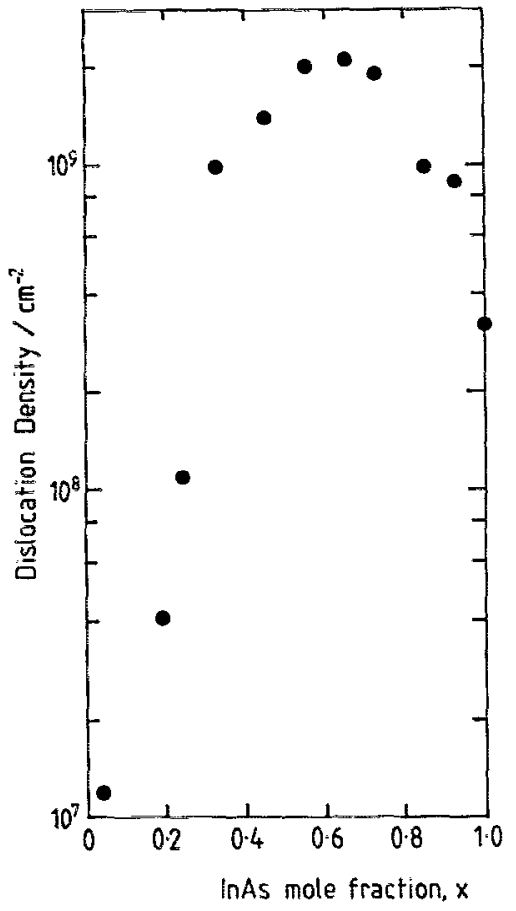

FIC. 2. Near-surface density of threading dislocations in $\operatorname{In}_{2}$ Ga $_{1 . . .}$ As samples plotted against InAs mole fraction, $x$.

As may he expected from Fig. 2, there exists a very broad maximum centered around $x \sim 0.5$, where the $\operatorname{In}_{x} \mathrm{Ga}_{1-x}$ As rocking curves are very wide $(\sim 900$ aresec $)$; towards the binary compositions these values become much smaller. The results are in agreement with those obtained by Fujita et al. ${ }^{15}$ for the same material system (although using a growth temperature of $480^{\circ} \mathrm{C}$ ) and, remarkably, those of Baribeau et al. ${ }^{16}$ working in the $\mathrm{Ge}_{x} \mathrm{Si}_{1-x} / \mathrm{Si}(100)$ system.

These observations may be compared with the growth mode observed by RHEED. This revealed that with increasing $x$, just beyond the point of relaxation the patterns appeared increasingly spotty (or 3D) but with streaks indicative of $2 \mathrm{D}$ growth remaining visible up to $x \sim 0.4 .^{11}$ In all cases, the regime of $3 \mathrm{D}$ or slightly $3 \mathrm{D}$ growth was always followed by $2 \mathrm{D}$ growth so that for all compositions well streaked RHEED features were always visible at the end of the growth.

\section{DISCUSSION AND CONCLUSIONS}

The equilibrium models of Van der Merwe $\mathrm{M}^{17}$ and Matthews and Blakeslee ${ }^{7}$ predict that pseudomorphic growth takes place until a critical thickness is reached. If the thickness of the growing film exceeds this limit, misfit dislocations are formed by glide of pre-existing dislocations. However, in carefully grown semiconductor crystals on lowdefect substrates, the number of available threading dislocations is generally not sufficient to accommodate the strain. Therefore new dislocations must nucleate. The effect of this process on the critical thickness has been calculated by People and Bean ${ }^{18}$ and in a more realistic ex- (a) $x=0.45$

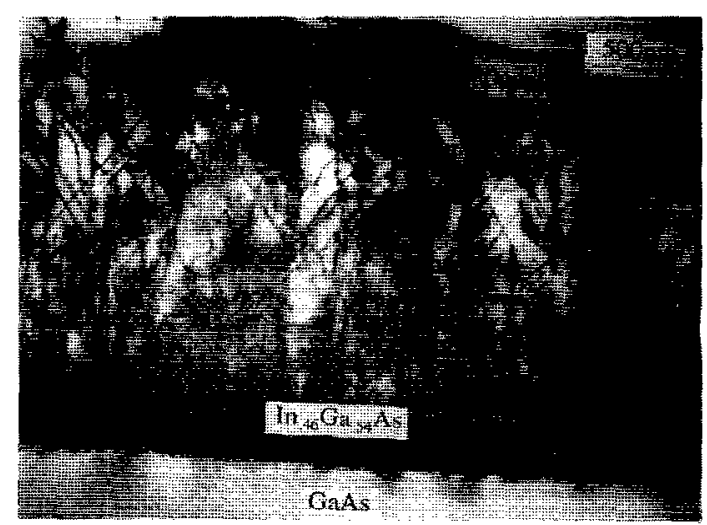

(b) $\mathrm{x}=1.0$

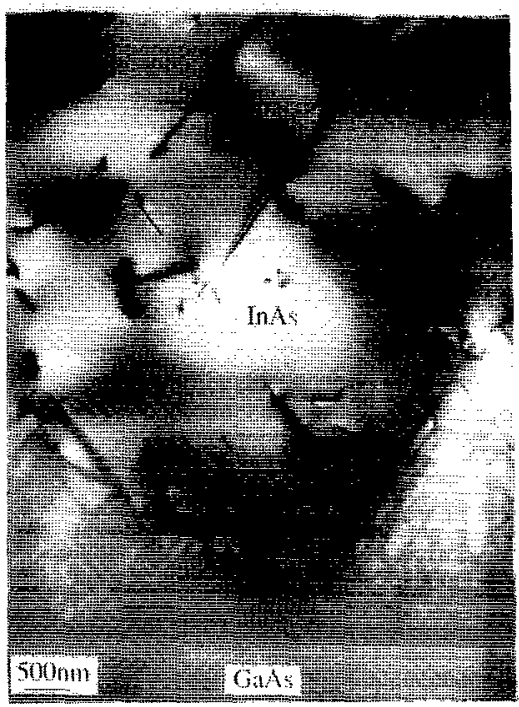

FIG. 3. Cross-sectional images of the $\mathrm{In}_{x} \mathrm{Ga}_{1} \mathrm{As} / \mathrm{GaAs}$ interface of samples: (a) $x=0.45$ and (b) $x=1.0$.

pression by Marée et al. ${ }^{19}$ The dislocations expected, and observed, to be formed in this process are $60^{\circ}$ mixed dislocations which glide along $\{111\}$ planes inclined to the interface.

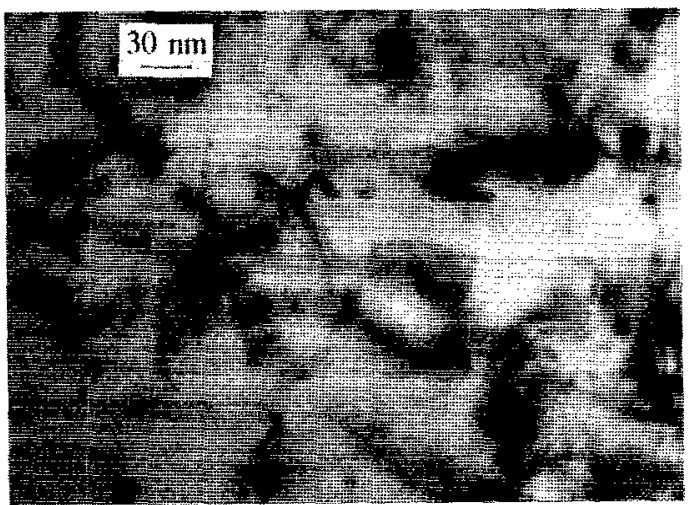

FIG. 4. Plan-view images of the edge dislocation network at the InAs/ GaAs interface. 


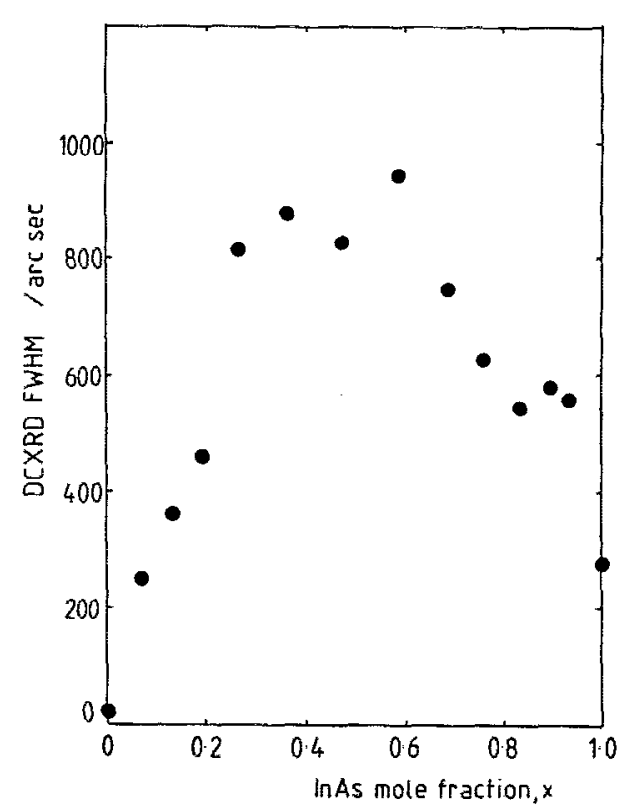

FIG. 5. Double-crystal $x$-ray diffraction FWHM of $\operatorname{In}_{x} \mathrm{Ga}_{1-x}$ As features plotted against InAs mole fraction, $x$.

The above provides an adequate basis for understanding the initial relaxation of low mismatched InGaAs on GaAs layers where 2D growth is maintained throughout. Further consideration of the microstructures of these films then allows the dislocation dynamics to be described in terms of the following sequence: ${ }^{20}$ nucleation of glissile half loops at the epilayer growth surface; glide and crystallographic realignment of these half loops to the interface on the four inclined $\{111\}$ variants; extension of the heterointerface dislocation segments along the two $\langle 011\rangle$ variants in the (100) plane to form simple orthogonal arrays; and, multiplication processes such as Hagen-Strunk multiplication $^{21}$ combined with appropriate dislocation reactions. Although $60^{\circ}$ dislocations dominate in thin, low mismatch films, edge dislocations are always present and become more apparent both during the final stages of relaxation, and especially as the mismatch is increased. ${ }^{1,3}$ These can be introduced, during this $2 \mathrm{D}$ growth, by the recombination of two $60^{\circ}$ dislocations or by climb processes. $^{13}$

At higher mole fractions, where relaxation is initially through the formation of islands commensurate with the substrate (i.e., 3D growth), an understanding of the problem is far less advanced. It has been noted that it is edge dislocations that dominate strain relief ${ }^{13}$ and it might be expected that these may be introduced either at the edges of islands or between islands as they coalesce, as a consequence of the unusually high stress concentrations in these regions. Observations have indeed indicated the formation of edge dislocations as a result of island coalescence, but while defects have also been reported at the edges of isolated islands (in $\mathrm{In}_{0.5} \mathrm{Ga}_{0.5} \mathrm{As}$ on $\mathrm{GaAs}{ }^{14}$ ), these have so far not been reported to be in the form of edge dislocations.

In our studies the TEM and DCXRD results have indicated that the degradation of the material quality, through an increase of the density of dislocations and DCXRD FWHM, is continuous up to $x \sim 0.5$. It has been found difficult to distinguish a distinct boundary between the so-called $2 \mathrm{D}$ growth mode at low mole fractions and the $3 \mathrm{D}$ mode either by these techniques, or by RHEED. The improvement in quality as $x$ increases from 0.5 is associated with networks of edge dislocations that become increasingly regular and which for InAs on GaAs is very regular indeed. This is, in many ways, the ideal situation since edge dislocations are the most efficient for relieving strain and are confined to the plane of the interface, i.e., they do not thread into the layer. It is then clear why the quality of thick InAs on GaAs films can be as good as thick $\mathrm{In}_{x} \mathrm{Ga}_{1-x}$ As $(x=0.1-0.2)$ on GaAs: in the latter case strain relief is by misfit dislocations (mainly $60^{\circ}$ dislocations) that are less efficient and thread into the epitaxial layer. The disparity in quality between the $\operatorname{In}_{0.5} \mathrm{Ga}_{0.5} \mathrm{As}$ and InAs films is presumably associated with a lack of coherence of the edge dislocation network in the former case. This leads to regions between the edge dislocations of high strain and the need for further defects. The more irregular the network the more additional defects that are required and introduced.

Unfortunately, the reason why regular networks of edge dislocations only occur at high InAs mole fractions is still unclear. One consideration is that as the misfit increases so do the shear stresses, and these may be capable of changing the nature of the network. However, this view is not supported by other experimental data. Comparing $\mathrm{Ge}_{x} \mathrm{Si}_{1-x}$ grown on $\mathrm{Si}$ with $\mathrm{In}_{x} \mathrm{Ga}_{1-x} \mathrm{As}$ deposited on GaAs their quality, as measured by DCXRD diffraction, is remarkably similar over the whole composition range (Ref. 11 and references therein), even though the misfits in the two cases are quite different (i.e., up to $4.2 \%$ and $7.2 \%$, respectively). Since in both cases the quality is worst at $x \sim 0.5$ it seems likely that alloy effects are also important. A clue may exist in the regularity of the misfit dislocations that form. It has previously been observed that the array of $60^{\circ}$ mixed dislocations that are very regular at low $x$ become very disordered between $x=0.25$ and $0.4 .^{22} \mathrm{Sim}$ ilarly here, a very regular array of edge dislocations is observed in InAs on GaAs films but rapidly becomes much less ordered as $x$ is decreased.

In summary, the poor quality of $\operatorname{In}_{x} \mathrm{Ga}_{1-x} \mathrm{As}$ grown on GaAs (100) substrates at compositions around $x=0.5$ can now be understood to result from networks of $60^{\circ}$ mixed dislocations at low $x$ and edge dislocations at high $x$ which become increasingly more irregular as $x=0.5$ is approached. In addition the magnitude of the misfit and the growth mode can no longer be invoked to explain all the characteristics of this system, instead other effects, perhaps alloy related, need to be considered.

\section{ACKNOWLEDGMENTS}

Two of us (D.I.W. and D.A.W.) gratefully acknowledge the financial assistance of the U. K. Science and Engineering Research Council (SERC) under the LowDimensional Structures and Devices Initiative. 
'G. C. Oshoum, J. Vac. Sci. Technol. B 4, 1423 (1986).

${ }^{2}$ R. F. Kopf, J. M. Kuo, J. Kovalchick, S. J. Pearton, E. D. Jones, and A. Ourmazd, J. Appl. Phys, 68, 4029 (1990).

${ }^{3} \mathrm{~K}$. Matsumura, D. Inoue, H. Nakano, M. Sawada, and Y. Harada, Jpn. J. Appl. Phys. 30, L166 (1991).

"M. C. Wu, N. A. Olsson, D. Sivco, and A. Y. Cho, Appl. Phys. Lett. 56, $221(1990)$.

${ }^{5}$ N. Chand, E. E. Becker, J. P. van der Ziel, S. N. G. Chu, and N. K. Dutta, Appl. Phys. Lett. 58, 1704 (1991).

${ }^{6}$ F. C, Frank and J. D. van der Merwe, Proc. R. Soc. (London) A 198, 216 (1949).

${ }^{7}$ J. W. Matthews and A. E. Blakeslee, J. Cryst. Growth 27, 118 (1974). 'J. Y. Yao, T. G. Anderson, and G. L. Dunlop, J. Appl. Phys. 69, 2224 (1991).

${ }^{9}$ A. M. Ceschin and J. Massies, J. Cryst. Growth 114, 693 (1991).

${ }^{10}$ B. Elman, E. S. Koteles, P. Melman, K. Ostreicher, and C. Sung, J. Appl. Phys. 70, 2634 (1991).

${ }^{11}$ D. I. Westwood, D. A. Woolf, and R. H. Williams, J. Cryst. Growth 98, $782(1989)$.
${ }^{12}$ J. Y. Yao, T. G. Andersson, and G. L. Dunlop, Appl. Phys. Lett. 53, 1420 (1988).

${ }^{13}$ K. H. Chang, P. K. Bhattacharya, and R. Gibala, J. Appl. Phys. 66, 2993 (1989).

${ }^{14}$ S. Guha, A. Madhukar, and K. C. Rajkumar, Appl. Phys. Lett. 57, 2110 (1990).

${ }^{15}$ S. Fujita, Y. Nakaoka, T. Uemura, M. Tabuchi, S. Noda, Y. Tadeka, and A. Sasaki, J. Cryst. Growth 95, 224 (1989).

${ }^{16}$ J. M. Baribeau, T. E. Jackman, D. C. Houghton, P. Maigné, and M. W. Denhoff, J. Appl. Phys. 63, 7538 (1988).

${ }^{17}$ J. H. van der Merwe, Surf. Sci. 31, 198 (1972).

${ }^{18}$ R. People and J. C. Bean. Appl. Phys. Lett. 47, 322 (1985).

${ }^{19}$ P. M. J. Marée, J. C. Barbour, J. F. van der Veen, K. L. Kavanagh, C. W. T. Bulle-Lieuwma, and M. P. A. Viegers, J. Appl. Phys. 62, 4413 (1987).

${ }^{20}$ M. N. Kableer, Phys. Rev. 131, 54 (1963).

${ }^{21}$ H. Strunk, W. Hagen, and E. Bauser, Appl. Phys. 18, 67 (1989).

${ }^{22}$ K. R. Breen, P. N. Uppal, and J. S. Ahearn, J. Vac. Sci. Technol. B 7, 758 (1989). 